\title{
Existence and uniqueness of positive solutions for fractional differential equations
}

Tao Zhu ${ }^{1 *}$

Correspondence:

zhutaoyzu@sina.cn

${ }^{1}$ Department of Mathematics and

Physics, Nanjing Institute of

Technology, Nanjing, P.R. China

\begin{abstract}
By some new integral inequalities of Henry-Gronwall type, we investigate the existence and uniqueness of positive solutions for fractional differential equations.

MSC: 39B62; 34A08; 26A33

Keywords: Fixed point theorem; Henry-Gronwall integral inequality; Fractional differential equation; Fractional derivative
\end{abstract}

\section{Introduction}

Fractional differential equations have gained considerable importance due to their applications in various sciences such as physics, mechanics, chemistry, engineering, etc. [1-7]. In the recent years, there has been a significant development in ordinary and partial differential equations involving fractional derivatives, see the monographs [8-10] and the papers in [11-17]. However, there have been few contributions to the existence and uniqueness of the following fractional differential equations:

$$
\left\{\begin{array}{l}
D_{c}^{\alpha} x(t)-D_{c}^{\beta} x(t)=f(t, x(t)), \quad t \in[0, T), 0<\beta<\alpha<1, \\
x(0)=x_{0} .
\end{array}\right.
$$

In most of the available literature, fractional integral inequalities play an important role in the qualitative analysis of the solutions for fractional differential equations (see [1417]). In this paper, by a method introduced by M. Medved' [18], we first study the following Henry-Gronwall integral inequalities:

$$
u(t) \leq a(t)+b_{1}(t) \int_{0}^{t}(t-s)^{\gamma_{1}-1} l_{1}(s) u(s) d s+b_{2}(t) \int_{0}^{t}(t-s)^{\gamma_{2}-1} l_{2}(s) u(s) d s
$$

and

$$
u(t) \leq a(t)+b_{1}(t) \int_{0}^{t}(t-s)^{\gamma_{1}-1} l_{1}(s) \varphi_{1}(u(s)) d s+b_{2}(t) \int_{0}^{t}(t-s)^{\gamma_{2}-1} l_{2}(s) \varphi_{2}(u(s)) d s,
$$

where $0<\gamma_{1}<\gamma_{2}<1$, which generalize the famous Henry inequalities [19]. Then using a suitable substitution, we construct an equivalent fractional integral equation of equation (1.1). By the above integral inequalities and fixed point theorem, we present the exis-

(c) The Author(s) 2019. This article is distributed under the terms of the Creative Commons Attribution 4.0 International License (http://creativecommons.org/licenses/by/4.0/), which permits unrestricted use, distribution, and reproduction in any medium, provided you give appropriate credit to the original author(s) and the source, provide a link to the Creative Commons license, and indicate if changes were made. 
tence and uniqueness of fractional differential equations (1.1). Finally, some examples are given to illustrate the applications of the obtained results.

\section{Preliminaries}

In this section, we introduce definitions and preliminary facts which are used throughout this paper.

Let $I=[0, a](0<a<+\infty)$ be a finite interval. $A C[0, a]$ is the space of functions which are absolutely continuous on $I \cdot L^{\infty}(0, a)$ is the space of measurable functions $f: I \rightarrow \Re$ with the norm $\|f\|_{L^{\infty}}=\inf \{c>0,|f(t)| \leq c$, a.e. $t \in I\} . C^{1}[0, a]$ is the space of functions which are continuously differentiable on $I$.

The Riemann-Liouville fractional integral and derivative of order $\alpha \in(0,1)$ are defined by

$$
I^{\alpha} x(t)=\frac{1}{\Gamma(\alpha)} \int_{0}^{t} \frac{x(s)}{(t-s)^{1-\alpha}} d s, \quad t>0
$$

and

$$
D^{\alpha} x(t)=\frac{1}{\Gamma(1-\alpha)} \frac{d}{d t} \int_{0}^{t} \frac{x(s)}{(t-s)^{\alpha}} d s, \quad t>0 .
$$

The Caputo fractional derivative of order $\alpha \in(0,1)$ is defined by

$$
D_{c}^{\alpha} x(t)=D^{\alpha} x(t)-\frac{x(0)}{\Gamma(1-\alpha)} t^{-\alpha}, \quad t>0 .
$$

In particular, when $x(t) \in A C[0, a]$,

$$
D_{c}^{\alpha} x(t)=\frac{1}{\Gamma(1-\alpha)} \int_{0}^{t} \frac{x^{\prime}(s)}{(t-s)^{\alpha}} d s, \quad t>0
$$

Lemma 2.1 ([8]) Let $\alpha \in(0,1)$ and $x \in L^{\infty}(0, a)$ or $x \in C[0, a]$, then

$$
\left(D_{c}^{\alpha} I^{\alpha} x\right)(t)=x(t) .
$$

Lemma $2.2([8])$ Let $\alpha \in(0,1)$ and $x \in A C[0, a]$ or $x \in C^{1}[0, a]$, then

$$
\left(I^{\alpha} D_{c}^{\alpha} x\right)(t)=x(t)-x(0)
$$

Theorem 2.3 Let $0<\beta<\alpha<1$ and $x \in A C[0, a]$ or $x \in C^{1}[0, a]$, then

$$
\left(D_{c}^{\alpha} I^{\alpha-\beta} x\right)(t)=D_{c}^{\beta} x(t)+\frac{x(0)}{\Gamma(1-\beta)} t^{-\beta} .
$$

Proof By Lemmas 2.1 and 2.2, we know

$$
\begin{aligned}
\left(D_{c}^{\alpha} I^{\alpha-\beta} x\right)(t) & =D_{c}^{\alpha} I^{\alpha-\beta}\left(\left(I^{\beta} D_{c}^{\beta} x\right)(t)+x(0)\right) \\
& =\left(D_{c}^{\alpha} I^{\alpha} D_{c}^{\beta} x\right)(t)+D_{c}^{\alpha} I^{\alpha-\beta}(x(0))
\end{aligned}
$$




$$
=D_{c}^{\beta} x(t)+\frac{x(0)}{\Gamma(1-\beta)} t^{-\beta}
$$

Theorem 2.4 Let $0<\beta<\alpha<1$ and $x=I^{\beta} \mu(t)$, where $\mu \in C[0, a]$, then

$$
\left(D_{c}^{\alpha} I^{\alpha-\beta} x\right)(t)=D_{c}^{\beta} x(t)
$$

Proof We know

$$
\begin{aligned}
\left(D_{c}^{\alpha} I^{\alpha-\beta} x\right)(t) & =\left(D_{c}^{\alpha} I^{\alpha-\beta} I^{\beta} \mu\right)(t) \\
& =\left(D_{c}^{\alpha} I^{\alpha} \mu\right)(t) \\
& =\mu(t) \\
& =D_{c}^{\beta} x(t) .
\end{aligned}
$$

Theorem 2.5 Let $0<\gamma_{1}<\gamma_{2}<1, a(t), b_{1}(t), b_{2}(t), l_{1}(t)$, and $l_{2}(t)$ be continuous, nonnegative functions on $[0,+\infty)$, and $u(t)$ be a continuous, nonnegative function on $[0,+\infty)$ with

$$
u(t) \leq a(t)+b_{1}(t) \int_{0}^{t}(t-s)^{\gamma_{1}-1} l_{1}(s) u(s) d s+b_{2}(t) \int_{0}^{t}(t-s)^{\gamma_{2}-1} l_{2}(s) u(s) d s
$$

Then the following assertions hold:

$$
\begin{aligned}
u(t) & \leq\left(3^{p-1} a^{p}(t)+3^{p-1} b^{p}(t)\left(A(t)+\int_{0}^{t} L(s) A(s) \exp \left(\int_{s}^{t} L(\tau) d \tau\right) d s\right)\right)^{\frac{1}{p}}, \\
t & \in[0,+\infty),
\end{aligned}
$$

where $b(t)=\max \left\{\frac{b_{1}(t) t^{\gamma_{1}-1+\frac{1}{q}}}{\left(q\left(\gamma_{1}-1\right)+1\right)^{\frac{1}{q}}}, \frac{b_{2}(t) t^{\gamma_{2}-1+\frac{1}{q}}}{\left(q\left(\gamma_{2}-1\right)+1\right)^{\frac{1}{q}}}\right\}, \quad A(t)=\int_{0}^{t} 3^{p-1}\left(l_{1}^{p}(s)+l_{2}^{p}(s)\right) a^{p}(s) d s, \quad L(t)=$ $3^{p-1} b^{p}(t)\left(l_{1}^{p}(t)+l_{2}^{p}(t)\right)$, and $p, q \in(1,+\infty)$ such that $\gamma_{1}+\frac{1}{q}>1$ and $\frac{1}{q}+\frac{1}{p}=1$.

Proof Choose nonnegative constants $p, q$ such that $\gamma_{1}+\frac{1}{q}>1$ and $\frac{1}{q}+\frac{1}{p}=1$. Using the Hölder inequality, we obtain

$$
\begin{aligned}
u(t) \leq & a(t)+b_{1}(t) \int_{0}^{t}(t-s)^{\gamma_{1}-1} l_{1}(s) u(s) d s \\
& +b_{2}(t) \int_{0}^{t}(t-s)^{\gamma_{2}-1} l_{2}(s) u(s) d s \\
\leq & a(t)+b_{1}(t)\left(\int_{0}^{t}(t-s)^{\left(\gamma_{1}-1\right) q} d s\right)^{\frac{1}{q}}\left(\int_{0}^{t}\left(l_{1}(s) u(s)\right)^{p} d s\right)^{\frac{1}{p}} \\
& +b_{2}(t)\left(\int_{0}^{t}(t-s)^{\left(\gamma_{2}-1\right) q} d s\right)^{\frac{1}{q}}\left(\int_{0}^{t}\left(l_{2}(s) u(s)\right)^{p} d s\right)^{\frac{1}{p}} \\
\leq & a(t)+\frac{b_{1}(t) t^{\gamma_{1}-1+\frac{1}{q}}}{\left(q\left(\gamma_{1}-1\right)+1\right)^{\frac{1}{q}}}\left(\int_{0}^{t} l_{1}^{p}(s) u^{p}(s) d s\right)^{\frac{1}{p}}
\end{aligned}
$$




$$
+\frac{b_{2}(t) t^{\gamma_{2}-1+\frac{1}{q}}}{\left(q\left(\gamma_{2}-1\right)+1\right)^{\frac{1}{q}}}\left(\int_{0}^{t} l_{2}^{p}(s) u^{p}(s) d s\right)^{\frac{1}{p}}
$$

Let $b(t)=\max \left\{\frac{b_{1}(t) t^{\gamma_{1}-1+\frac{1}{q}}}{\left(q\left(\gamma_{1}-1\right)+1\right)^{\frac{1}{q}}}, \frac{b_{2}(t) t^{\gamma_{2}-1+\frac{1}{q}}}{\left(q\left(\gamma_{2}-1\right)+1\right)^{\frac{1}{q}}}\right\}$. Then

$$
u^{p}(t) \leq 3^{p-1} a^{p}(t)+3^{p-1} b^{p}(t) \int_{0}^{t}\left(l_{1}^{p}(s)+l_{2}^{p}(s)\right) u^{p}(s) d s
$$

and

$$
\begin{aligned}
& \int_{0}^{t}\left(l_{1}^{p}(s)+l_{2}^{p}(s)\right) u^{p}(s) d s \\
& \quad \leq \int_{0}^{t} 3^{p-1}\left(l_{1}^{p}(s)+l_{2}^{p}(s)\right) a^{p}(s) d s \\
& \quad+\int_{0}^{t} 3^{p-1} b^{p}(s)\left(l_{1}^{p}(s)+l_{2}^{p}(s)\right) \int_{0}^{s}\left(l_{1}^{p}(\tau)+l_{2}^{p}(\tau)\right) u^{p}(\tau) d \tau d s .
\end{aligned}
$$

Let $w(t)=\int_{0}^{t}\left(l_{1}^{p}(s)+l_{2}^{p}(s)\right) u^{p}(s) d s, A(t)=\int_{0}^{t} 3^{p-1}\left(l_{1}^{p}(s)+l_{2}^{p}(s)\right) a^{p}(s) d s$, and $L(t)=3^{p-1} b^{p}(t) \times$ $\left(l_{1}^{p}(t)+l_{2}^{p}(t)\right)$. Then

$$
w(t) \leq A(t)+\int_{0}^{t} L(s) w(s) d s
$$

By Gronwall's integral inequality, we have

$$
w(t) \leq A(t)+\int_{0}^{t} L(s) A(s) \exp \left(\int_{s}^{t} L(\tau) d \tau\right) d s
$$

By (2.6) and (2.9) we obtain inequality (2.4) and complete the proof.

Theorem 2.6 Let $0<\gamma_{1}<\gamma_{2}<1, a(t), b_{1}(t), b_{2}(t), l_{1}(t)$, and $l_{2}(t)$ be nondecreasing, nonnegative, and continuous functions on $[0, T)(0<T \leq+\infty), \varphi_{1}, \varphi_{2}:[0,+\infty) \rightarrow[0,+\infty)$ be continuous, nondecreasing functions, and $u(t)$ be a continuous, nonnegative function on $[0, T)$ with

$$
\begin{aligned}
u(t) \leq & a(t)+b_{1}(t) \int_{0}^{t}(t-s)^{\gamma_{1}-1} l_{1}(s) \varphi_{1}(u(s)) d s \\
& +b_{2}(t) \int_{0}^{t}(t-s)^{\gamma_{2}-1} l_{2}(s) \varphi_{2}(u(s)) d s
\end{aligned}
$$

Then

$$
u(t) \leq\left(\Omega^{-1}\left(\Omega(A(t))+B_{1}(t) \int_{0}^{t} l_{1}^{p}(s) d s+B_{2}(t) \int_{0}^{t} l_{2}^{p}(s) d s\right)\right)^{\frac{1}{p}}, \quad t \in\left[0, T_{1}\right]
$$

where $A(t)=3^{p-1} a^{p}(t), \quad B_{1}(t)=3^{p-1}\left(\frac{b_{1}(t) t^{\gamma_{1}-1+\frac{1}{q}}}{\left(q\left(\gamma_{1}-1\right)+1\right)^{\frac{1}{q}}}\right)^{p}, \quad B_{2}(t)=3^{p-1}\left(\frac{b_{2}(t) t^{\gamma_{2}-1+\frac{1}{q}}}{\left(q\left(\gamma_{2}-1\right)+1\right)^{\frac{1}{q}}}\right)^{p}, \quad \Omega(x)=$ $\int_{t_{0}}^{x} \frac{1}{\mu_{1}(t)+\mu_{2}(t)} d t, \mu_{1}(t)=\varphi_{1}^{p}\left(t^{\frac{1}{p}}\right), \mu_{2}(t)=\varphi_{2}^{p}\left(t^{\frac{1}{p}}\right), t_{0}>0, \Omega^{-1}$ is the inverse of $\Omega$, and $T_{1} \in$ 
$(0, T)$ is such that $\Omega(A(t))+B_{1}(t) \int_{0}^{t} l_{1}^{p}(s) d s+B_{2}(t) \int_{0}^{t} l_{2}^{p}(s) d s \in \operatorname{Dom}\left(\Omega^{-1}\right)$ for all $t \in\left[0, T_{1}\right]$, and $p, q \in(1,+\infty)$ such that $\gamma_{1}+\frac{1}{q}>1$ and $\frac{1}{q}+\frac{1}{p}=1$.

Proof Choose nonnegative constants $p, q$ such that $\gamma_{1}+\frac{1}{q}>1$ and $\frac{1}{q}+\frac{1}{p}=1$. Using the Hölder inequality, we obtain

$$
\begin{aligned}
u(t) \leq & a(t)+b_{1}(t) \int_{0}^{t}(t-s)^{\gamma_{1}-1} l_{1}(s) \varphi_{1}(u(s)) d s \\
& +b_{2}(t) \int_{0}^{t}(t-s)^{\gamma_{2}-1} l_{2}(s) \varphi_{2}(u(s)) d s \\
\leq & a(t)+\frac{b_{1}(t) t^{\gamma_{1}-1+\frac{1}{q}}}{\left(q\left(\gamma_{1}-1\right)+1\right)^{\frac{1}{q}}}\left(\int_{0}^{t} l_{1}^{p}(s) \varphi_{1}^{p}(u(s)) d s\right)^{\frac{1}{p}} \\
& +\frac{b_{2}(t) t^{\gamma_{2}-1+\frac{1}{q}}}{\left(q\left(\gamma_{2}-1\right)+1\right)^{\frac{1}{q}}}\left(\int_{0}^{t} l_{2}^{p}(s) \varphi_{2}^{p}(u(s)) d s\right)^{\frac{1}{p}} .
\end{aligned}
$$

Then

$$
\begin{aligned}
u^{p}(t) \leq & 3^{p-1} a^{p}(t)+3^{p-1}\left(\frac{b_{1}(t) t^{\gamma_{1}-1+\frac{1}{q}}}{\left(q\left(\gamma_{1}-1\right)+1\right)^{\frac{1}{q}}}\right)^{p} \int_{0}^{t} l_{1}^{p}(s) \varphi_{1}^{p}(u(s)) d s \\
& +3^{p-1}\left(\frac{b_{2}(t) t^{\gamma_{2}-1+\frac{1}{q}}}{\left(q\left(\gamma_{2}-1\right)+1\right)^{\frac{1}{q}}}\right)^{p} \int_{0}^{t} l_{2}^{p}(s) \varphi_{2}^{p}(u(s)) d s .
\end{aligned}
$$

Let $w(t)=u^{p}(t), A(t)=3^{p-1} a^{p}(t), B_{1}(t)=3^{p-1}\left(\frac{b_{1}(t) t^{\gamma_{1}-1+\frac{1}{q}}}{\left(q\left(\gamma_{1}-1\right)+1\right)^{\frac{1}{q}}}\right)^{p}$, and $B_{2}(t)=3^{p-1}\left(\frac{b_{2}(t) t^{\gamma_{2}-1+\frac{1}{q}}}{\left(q\left(\gamma_{2}-1\right)+1\right)^{\frac{1}{q}}}\right)^{p}$. Fix any $T_{0} \in\left[0, T_{1}\right]$, then for $t \in\left[0, T_{0}\right]$ and (2.13) we have

$$
\begin{aligned}
w(t) \leq & A\left(T_{0}\right)+B_{1}\left(T_{0}\right) \int_{0}^{t} l_{1}^{p}(s) \mu_{1}(w(s)) d s \\
& +B_{2}\left(T_{0}\right) \int_{0}^{t} l_{2}^{p}(s) \mu_{2}(w(s)) d s .
\end{aligned}
$$

Let $V(t)=A\left(T_{0}\right)+B_{1}\left(T_{0}\right) \int_{0}^{t} l_{1}^{p}(s) \mu_{1}(w(s)) d s+B_{2}\left(T_{0}\right) \int_{0}^{t} l_{2}^{p}(s) \mu_{2}(w(s)) d s$, then we get

$$
\begin{aligned}
V^{\prime}(t) & =B_{1}\left(T_{0}\right) l_{1}^{p}(t) \mu_{1}(w(t))+B_{2}\left(T_{0}\right) l_{2}^{p}(t) \mu_{2}(w(t)) \\
& \leq B_{1}\left(T_{0}\right) l_{1}^{p}(t) \mu_{1}(V(t))+B_{2}\left(T_{0}\right) l_{2}^{p}(t) \mu_{2}(V(t)) .
\end{aligned}
$$

This yields

$$
\frac{V^{\prime}(t)}{\mu_{1}(V(t))+\mu_{2}(V(t))} \leq B_{1}\left(T_{0}\right) l_{1}^{p}(t)+B_{2}\left(T_{0}\right) l_{2}^{p}(t)
$$

or

$$
\frac{d}{d t} \Omega(V(t)) \leq B_{1}\left(T_{0}\right) l_{1}^{p}(t)+B_{2}\left(T_{0}\right) l_{2}^{p}(t) .
$$


Integrating this inequality from 0 to $t \in\left[0, T_{0}\right]$, we obtain

$$
\Omega(V(t)) \leq \Omega\left(A\left(T_{0}\right)\right)+\int_{0}^{t} B_{1}\left(T_{0}\right) l_{1}^{p}(s)+B_{2}\left(T_{0}\right) l_{2}^{p}(s) d s
$$

then

$$
V(t) \leq \Omega^{-1}\left(\Omega\left(A\left(T_{0}\right)\right)+\int_{0}^{t} B_{1}\left(T_{0}\right) l_{1}^{p}(s)+B_{2}\left(T_{0}\right) l_{2}^{p}(s) d s\right), \quad t \in\left[0, T_{0}\right]
$$

and

$$
u(t) \leq\left(\Omega^{-1}\left(\Omega\left(A\left(T_{0}\right)\right)+\int_{0}^{t} B_{1}\left(T_{0}\right) l_{1}^{p}(s)+B_{2}\left(T_{0}\right) l_{2}^{p}(s) d s\right)\right)^{\frac{1}{p}}, \quad t \in\left[0, T_{0}\right]
$$

So

$$
u\left(T_{0}\right) \leq\left(\Omega^{-1}\left(\Omega\left(A\left(T_{0}\right)\right)+B_{1}\left(T_{0}\right) \int_{0}^{T_{0}} l_{1}^{p}(s) d s+B_{2}\left(T_{0}\right) \int_{0}^{T_{0}} l_{2}^{p}(s) d s\right)\right)^{\frac{1}{p}} .
$$

Now replacing $T_{0}$ by $t$ in inequality (2.21), we obtain the result (2.11) valid for $t \in\left[0, T_{1}\right]$ provided

$$
\Omega(A(t))+B_{1}(t) \int_{0}^{t} l_{1}^{p}(s) d s+B_{2}(t) \int_{0}^{t} l_{2}^{p}(s) d s \in \operatorname{Dom}\left(\Omega^{-1}\right)
$$

for all $t \in\left[0, T_{1}\right]$.

Lemma $2.7([20,21])$ Let $E$ be a Banach space $X, C$ be a closed, convex subset of $E, U$ be an open subset of $C$, and $P \in U$. Suppose that $F: \bar{U} \rightarrow C$ is a continuous, compact map. Then either

(a) F has a fixed point in $\bar{U}$; or

(b) there are $u \in \partial U$ (the boundary of $U$ in $C$ ) and $\lambda \in(0,1)$ with $u=\lambda F(u)+(1-\lambda) P$.

Lemma 2.8 ([20, 21]) Let E be a Hausdorff locally convex linear topological space, $C$ be a convex subset of $E, U$ be an open subset of $C$, and $P \in U$. Suppose that $F: \bar{U} \rightarrow C$ is a continuous, compact map. Then either

(a) F has a fixed point in $\bar{U}$; or

(b) there are $u \in \partial U$ (the boundary of $U$ in $C$ ) and $\lambda \in(0,1)$ with $u=\lambda F(u)+(1-\lambda) P$.

\section{Main results}

In this section, we give the existence and uniqueness results of the fractional differential equations (1.1).

Theorem 3.1 $f: \Re^{+} \times \Re \rightarrow \Re$ is a continuous function. If $x(\cdot) \in C[0, a]$ is the solution of the following integral equation

$$
x(t)=x_{0}+\frac{1}{\Gamma(\alpha-\beta)} \int_{0}^{t}(t-s)^{\alpha-\beta-1}\left(x(s)-x_{0}\right) d s+\frac{1}{\Gamma(\alpha)} \int_{0}^{t}(t-s)^{\alpha-1} f(s, x(s)) d s,
$$

then $x(t)$ is the solution of the fractional integral equation (1.1). 
Proof If $x(t) \in C[0, a]$ is the solution of the integral equation (3.1), we know $x(0)=x_{0}$ and

$$
x(t)-x_{0}=I^{\alpha-\beta}\left(x(t)-x_{0}\right)+I^{\alpha} f(t, x(t))=I^{\alpha-\beta} \mu(t),
$$

where $\mu(t)=x(t)-x_{0}+I^{\beta} f(t, x(t))$. By (3.1) and (3.2), we obtain

$$
x(t)-x_{0}=I^{\alpha-\beta}\left(x(t)-x_{0}\right)+I^{\alpha} f(t, x(t))=I^{2(\alpha-\beta)} \mu(t)+I^{\alpha} f(t, x(t)) .
$$

If $2(\alpha-\beta)<\alpha$, then $x(t)-x_{0}=I^{2(\alpha-\beta)} \mu_{1}(t)$, where $\mu_{1}(t)=\mu(t)+I^{2 \beta-\alpha} f(t, x(t))$. By the same step, we obtain $x(t)-x_{0} \in I^{\alpha} \phi_{1}(t)$ and $x(t)-x_{0} \in I^{\beta} \phi_{2}(t)$, where $\phi_{1}(t), \phi_{2}(t) \in C[0, a]$.

By Lemma 2.1 and Theorem 2.4, we get

$$
\begin{aligned}
D_{c}^{\alpha} x(t) & =D_{c}^{\alpha} I^{\alpha-\beta}\left(x(t)-x_{0}\right)+D_{c}^{\alpha} I^{\alpha} f(t, x(t)) \\
& =D_{c}^{\beta}\left(x(t)-x_{0}\right)+f(t, x(t)) \\
& =D_{c}^{\beta} x(t)+f(t, x(t)) .
\end{aligned}
$$

Theorem 3.2 Let $x_{0}>0, f: \Re^{+} \times \Re^{+} \rightarrow \Re^{+}$be a continuous function, and there exist nonnegative continuous functions $l(t)$ and $k(t)$ such that

$$
|f(t, x)| \leq l(t)|x|+k(t)
$$

for all $x \in \Re^{+}, t \in[0, \infty)$. Then equation (1.1) has at least one positive solution on $[0, \infty)$.

Proof Consider the operator $G: W \rightarrow W$ defined by

$$
\begin{aligned}
(G x)(t)= & x_{0}+\frac{1}{\Gamma(\alpha-\beta)} \int_{0}^{t}(t-s)^{\alpha-\beta-1}\left(x(s)-x_{0}\right) d s \\
& +\frac{1}{\Gamma(\alpha)} \int_{0}^{t}(t-s)^{\alpha-1} f(s, x(s)) d s,
\end{aligned}
$$

where $W=\left\{x(t) \in C[0,+\infty) \mid x(t) \geq x_{0}\right\}$.

By Theorem 3.1, we know that the fixed points of operator $G$ are solutions of equation (1.1). We can show that $G: W \rightarrow W$ is continuous and compact by the usual techniques (see $[12,13])$.

Let $U=\left\{x \in W:|x(t)|<\left(3^{p-1} a^{p}(t)+3^{p-1} b^{p}(t)\left(A(t)+\int_{0}^{t} L(s) A(s) \exp \left(\int_{s}^{t} L(\tau) d \tau\right) d s\right)\right)^{\frac{1}{p}}+\right.$ $1, t \in[0, \infty)\}$, where $a(t)=\left|x_{0}\right|+\left|\frac{x_{0} t^{\alpha-\beta}}{(\alpha-\beta) \Gamma(\alpha-\beta)}\right|+\frac{1}{\Gamma(\alpha)} \frac{t^{\alpha-1+\frac{1}{q}}}{((\alpha-1) q+1)^{\frac{1}{q}}}\left(\int_{0}^{t} k^{p}(s) d s\right)^{\frac{1}{p}}, \quad b(t)=$ $\max \left\{\frac{\frac{1}{\Gamma(\alpha-\beta)} t^{\alpha-\beta-1+\frac{1}{q}}}{(q(\alpha-\beta-1)+1)^{\frac{1}{q}}}, \frac{\frac{1}{\Gamma(\alpha)} t^{\alpha-1+\frac{1}{q}}}{(q(\alpha-1)+1)^{\frac{1}{q}}}\right\}, A(t)=\int_{0}^{t} 3^{p-1}\left(1+l^{p}(s)\right) a^{p}(s) d s, L(t)=3^{p-1} b^{p}(t)\left(1+l^{p}(t)\right)$, and $p, q \in(1,+\infty)$ such that $\alpha-\beta+\frac{1}{q}>1$ and $\frac{1}{q}+\frac{1}{p}=1$.

If $x \in W$ is any solution of

$$
\begin{aligned}
x(t)= & (1-\lambda) x_{0}+\lambda\left(\frac{1}{\Gamma(\alpha-\beta)} \int_{0}^{t}(t-s)^{\alpha-\beta-1}\left(x(s)-x_{0}\right) d s\right. \\
& \left.+\frac{1}{\Gamma(\alpha)} \int_{0}^{t}(t-s)^{\alpha-1} f(s, x(s)) d s\right)
\end{aligned}
$$

for $\lambda \in(0,1)$. 
Then

$$
\begin{aligned}
|x(t)| \leq & \left|x_{0}\right|+\left|\frac{x_{0} t^{\alpha-\beta}}{(\alpha-\beta) \Gamma(\alpha-\beta)}\right| \\
& \left.+\left|\frac{1}{\Gamma(\alpha-\beta)} \int_{0}^{t}(t-s)^{\alpha-\beta-1} x(s) d s\right|+\mid \frac{1}{\Gamma(\alpha)} \int_{0}^{t}(t-s)^{\alpha-1} f(s, x(s)) d s\right) \mid \\
\leq & \left|x_{0}\right|+\left|\frac{x_{0} t^{\alpha-\beta}}{(\alpha-\beta) \Gamma(\alpha-\beta)}\right|+\frac{1}{\Gamma(\alpha)} \frac{t^{\alpha-1+\frac{1}{q}}}{((\alpha-1) q+1)^{\frac{1}{q}}}\left(\int_{0}^{t} k^{p}(s) d s\right)^{\frac{1}{p}} \\
& +\frac{1}{\Gamma(\alpha-\beta)} \int_{0}^{t}(t-s)^{\alpha-\beta-1}|x(s)| d s+\frac{1}{\Gamma(\alpha)} \int_{0}^{t}(t-s)^{\alpha-1} l(s)|x(s)| d s .
\end{aligned}
$$

Consequently, by Theorem 2.5, we can get

$$
\begin{aligned}
& |x(t)| \leq\left(3^{p-1} a^{p}(t)+3^{p-1} b^{p}(t)\left(A(t)+\int_{0}^{t} L(s) A(s) \exp \left(\int_{s}^{t} L(\tau) d \tau\right) d s\right)\right)^{\frac{1}{p}}, \\
& \quad t \in[0, \infty) .
\end{aligned}
$$

Applying Lemma 2.8, we can obtain that $G$ has at least one fixed point in $W$. Thus, the proof is completed.

Theorem 3.3 Iff : $\mathfrak{R}^{+} \times \mathfrak{R}^{+} \rightarrow \mathfrak{R}^{+}$is a continuous function and

$$
|f(t, x)-f(t, y)| \leq l(t)|x-y|
$$

for all $x, y \in \mathfrak{R}^{+}$and $t \in[0,+\infty)$, where nonnegative function $l(t) \in C[0,+\infty)$, then equation (1.1) has a unique positive solution on $[0,+\infty)$.

Proof By Theorem 3.2, we suppose that $x_{1}(t), x_{2}(t)$ are two positive solutions of equation (1.1). Then

$$
\begin{aligned}
\left|x_{1}(t)-x_{2}(t)\right| \leq & \left|\frac{1}{\Gamma(\alpha-\beta)} \int_{0}^{t}(t-s)^{\alpha-\beta-1}\left(x_{1}(s)-x_{2}(s)\right) d s\right| \\
& +\left|\frac{1}{\Gamma(\alpha)} \int_{0}^{t}(t-s)^{\alpha-1}\left(f\left(s, x_{1}(s)\right)-f\left(s, x_{2}(s)\right)\right) d s\right| \\
\leq & \frac{1}{\Gamma(\alpha-\beta)} \int_{0}^{t}(t-s)^{\alpha-\beta-1}\left|x_{1}(s)-x_{2}(s)\right| d s \\
& \left.+\frac{1}{\Gamma(\alpha)} \int_{0}^{t}(t-s)^{\alpha-1} l(s) \mid x_{1}(s)\right)-x_{2}(s) \mid d s .
\end{aligned}
$$

By Theorem 2.5, we can get $x_{1}(t)=x_{2}(t)$.

Theorem 3.4 Let $x_{0}>0, f:[0, T] \times \Re^{+} \rightarrow \Re^{+}$be a continuous function, and there exist a nonnegative function $l(t) \in C[0, T]$ and a nonnegative nondecreasing function $\omega \in$ $C[0,+\infty)$ such that

$$
|f(t, x)| \leq l(t) \omega(|x|)
$$


Then the initial value problem (1.1) has at least a continuous positive solution on $[0, T]$ provided that

$$
\Omega(A(t))+t B_{1}(t)+B_{2}(t) \int_{0}^{t} l^{p}(s) d s \in \operatorname{Dom}\left(\Omega^{-1}\right)
$$

for all $t \in[0, T]$, where $A(t)=3^{p-1}\left(\left|x_{0}\right|+\frac{\left|x_{0} t^{\alpha-\beta}\right|}{(\alpha-\beta) \Gamma(\alpha-\beta)}\right)^{p}, B_{1}(t)=3^{p-1}\left(\frac{\frac{1}{\Gamma(\alpha-\beta)} t^{\alpha-\beta-1+\frac{1}{q}}}{(q(\alpha-\beta-1)+1)^{\frac{1}{q}}}\right)^{p}, B_{2}(t)=$ $3^{p-1}\left(\frac{\frac{1}{\Gamma(\alpha)} t^{\alpha-1+\frac{1}{q}}}{(q(\alpha-1)+1)^{\frac{1}{q}}}\right)^{p}, \Omega(x)=\int_{t_{0}}^{x} \frac{1}{\mu_{1}(t)+\mu_{2}(t)} d t, \mu_{1}(t)=t, \mu_{2}(t)=\omega^{p}\left(t^{\frac{1}{p}}\right), t_{0}>0, \Omega^{-1}$ is the inverse of $\Omega$, and $p, q \in(1,+\infty)$ such that $\alpha-\beta+\frac{1}{q}>1$ and $\frac{1}{q}+\frac{1}{p}=1$.

Proof Consider the operator $G: W \rightarrow W$ defined by

$$
\begin{aligned}
(G x)(t)= & x_{0}+\frac{1}{\Gamma(\alpha-\beta)} \int_{0}^{t}(t-s)^{\alpha-\beta-1}\left(x(s)-x_{0}\right) d s \\
& +\frac{1}{\Gamma(\alpha)} \int_{0}^{t}(t-s)^{\alpha-1} f(s, x(s)) d s,
\end{aligned}
$$

where $W=\left\{x \in C[0, T] \mid x(t) \geq x_{0}\right\}$.

Similarly with the proof of Theorem 3.2 , we can show that $G: W \rightarrow W$ is continuous and compact.

Let $U=\left\{x \in W:|x(t)|<\left(\Omega^{-1}\left(\Omega(A(t))+t B_{1}(t)+B_{2}(t) \int_{0}^{t} l^{p}(s) d s\right)\right)^{\frac{1}{p}}+1, t \in[0, T]\right\}$.

If $x \in W$ is any solution of

$$
\begin{aligned}
x(t)= & (1-\lambda) x_{0}+\lambda\left(\frac{1}{\Gamma(\alpha-\beta)} \int_{0}^{t}(t-s)^{\alpha-\beta-1}\left(x(s)-x_{0}\right) d s\right. \\
& \left.+\frac{1}{\Gamma(\alpha)} \int_{0}^{t}(t-s)^{\alpha-1} f(s, x(s)) d s\right)
\end{aligned}
$$

for $\lambda \in(0,1)$.

Then

$$
\begin{aligned}
|x(t)| \leq & \left|x_{0}\right|+\frac{\left|x_{0} t^{\alpha-\beta}\right|}{(\alpha-\beta) \Gamma(\alpha-\beta)} \\
& +\frac{1}{\Gamma(\alpha-\beta)} \int_{0}^{t}(t-s)^{\alpha-\beta-1}|x(s)| d s \\
& +\frac{1}{\Gamma(\alpha)} \int_{0}^{t}(t-s)^{\alpha-1} l(s) \omega(|x(s)|) d s .
\end{aligned}
$$

By Theorem 2.6, we can get

$$
|x(t)| \leq\left(\Omega^{-1}\left(\Omega(A(t))+t B_{1}(t)+B_{2}(t) \int_{0}^{t} l^{p}(s) d s\right)\right)^{\frac{1}{p}}, \quad t \in[0, T]
$$

By Lemma 2.7, $G$ has at least one fixed point in $W$. Thus, the proof is completed. 


\section{Examples}

Example 4.1

$$
\left\{\begin{array}{l}
D_{c}^{\frac{1}{2}} x(t)-D_{c}^{\frac{1}{4}} x(t)=t^{2} x^{\frac{1}{2}}(t) \\
x(0)=1
\end{array}\right.
$$

We know $\left|t^{2} x^{\frac{1}{2}}(t)\right| \leq \frac{t^{2}(|x(t)|+1)}{2}$, all assumptions of Theorem 3.2 are satisfied. Hence equation (4.1) has at least one positive solution on $[0,+\infty)$.

\section{Example 4.2}

$$
\left\{\begin{array}{l}
D_{c}^{\frac{1}{2}} x(t)-D_{c}^{\frac{1}{4}} x(t)=e^{t} \ln (1+x(t)), \\
x(0)=1 .
\end{array}\right.
$$

We know $|\ln (1+x)-\ln (1+y)| \leq|x-y|$ for all $x, y \in(0,+\infty)$. From Theorem 3.3, equation (4.2) has a unique positive solution on $[0,+\infty)$.

\section{Example 4.3}

$$
\left\{\begin{array}{l}
D_{c}^{\frac{1}{2}} x(t)-D_{c}^{\frac{1}{4}} x(t)=t x^{2}(t) \\
x(0)=1
\end{array}\right.
$$

Let $q=\frac{5}{4}$ and $p=5$, from Theorem 3.4, equation (4.3) has at least one positive solution on $[0, T]$ provided that

$$
\begin{aligned}
& \ln \left(3^{4}\left(1+\frac{4 T^{\frac{1}{4}}}{\Gamma\left(\frac{1}{4}\right)}\right)^{5}\right)+3^{4}\left(\frac{16^{\frac{4}{5}} T^{\frac{1}{20}}}{\Gamma\left(\frac{1}{4}\right)}\right)^{5} T+3^{4}\left(\frac{8^{\frac{4}{5}} T^{\frac{3}{10}}}{3^{\frac{4}{5}} \Gamma\left(\frac{1}{2}\right)}\right)^{5} \frac{T^{6}}{6} \\
& \quad<\ln \left(1+3^{4}\left(1+\frac{4 T^{\frac{1}{4}}}{\Gamma\left(\frac{1}{4}\right)}\right)^{5}\right)
\end{aligned}
$$

\section{Acknowledgements}

The author would like to thank the handling editors and the anonymous reviewers.

Funding

This work was supported by the funding (No: CKJB201508; CKJB201709) from Nanjing Institute of Technology.

Availability of data and materials

Not applicable.

Competing interests

The author declares that he has no competing interests.

Authors' contributions

The author conceived of the study, drafted the manuscript, and approved the final manuscript.

\section{Publisher's Note}

Springer Nature remains neutral with regard to jurisdictional claims in published maps and institutional affiliations.

Received: 17 October 2018 Accepted: 23 January 2019 Published online: 30 January 2019 


\section{References}

1. Diethelm, K., Freed, A.D.: On the solution of nonlinear fractional order differential equations used in the modelling of viscoplasticty. In: Keil, F., Mackens, W., Voss, H., Werther, J. (eds.) Scientific Computing in Chemical Engineering II-Computational Fluid Dynamics, Reaction Engineering and Molecular Properties, pp. 217-224. Springer, Heidelberg (1999)

2. Gaul, L., Klein, P., Kempfle, S.: Damping description involving fractional operators. Mech. Syst. Signal Process. 5, 81-88 (1991)

3. Glockle, W.G., Nonnenmacher, T.F.: A fractional calculus approach of self-similar protein dynamics. Biophys. J 68, 46-53 (1995)

4. Hilfer, R.: Applications of Fractional Calculus in Physics. World Scientific, Singapore (2000)

5. Mainardi, F.: Fractional calculus: some basic problems in continuum and statistical mechanics. In: Carpinteri, A., Mainardi, F. (eds.) Fractals and Fractional Calculus in Continuum Mechanics, pp. 291-348. Springer, Wien (1997)

6. Metzler, F., Schick, W., Kilian, H.G., Nonnenmacher, T.F.: Relaxation in filled polymers: a fractional calculus approach. J. Chem. Phys. 103, 7180-7186 (1995)

7. Oldham, K.B., Spanier, J.: The Fractional Calculus. Academic Press, New York (1974)

8. Kilbas, A.A., Srivastava, H.M., Trujillo, J.J.: Theory and Applications of Fractional Differential Equations. North-Holland Mathematics Studies, vol. 204. Elsevier, Amsterdam (2006)

9. Podlubny, I.: Fractional Differential Equations: An Introduction to Fractional Derivatives, Fractional Differential Equations, to Methods of Their Solution and Some of Their Applications. Academic Press, San Diego (1999)

10. Miller, K.S., Ross, B.: An Introduction to the Fractional Calculus and Differential Equations. Wiley, New York (1993)

11. Lin, W.: Global existence theory and chaos control of fractional differential equations. J. Math. Anal. Appl. 332, 709-726 (2007)

12. Zhou, Y.: Existence and uniqueness of solutions for a system of fractional differential equations. Fract. Calc. Appl. Anal. $12,195-204(2009)$

13. Wang, J., Zhou, Y., Fečkan, M.: Abstract Cauchy problem for fractional differential equations. Nonlinear Dyn. 71, 685-700 (2013)

14. Zhu, T.: New Henry-Gronwall integral inequalities and their applications to fractional differential equations. Bull. Braz. Math. Soc. 49, 647-657 (2018)

15. Ye, H., Gao, J., Ding, Y.: A generalized Gronwall inequality and its application to a fractional differential equation. J. Math. Anal. Appl. 328, 1075-1081 (2007)

16. Denton, Z., Vatsala, A.S.: Fractional integral inequalities and applications. Comput. Math. Appl. 59, 1087-1094 (2010)

17. Ma, Q., Pečarić, J.: Some new explicit bounds for weakly singular integral inequalities with applications to fractional differential equations and integral equations. J. Math. Anal. Appl. 341, 894-905 (2008)

18. Medved', M.: A new approach to an analysis of Henry type integral inequalities and their bihair type versions. J. Math. Anal. Appl. 214, 349-366 (1997)

19. Henry, D.: Geometric Theory of Semilinear Parabolic Equations. Springer, Berlin (1981)

20. Agarwal, R.P., Meehan, M., O'Regan, D.: Fixed Point Theory and Applications. Cambridge University Press, Cambridge (2001)

21. Granas, A., Dugundji, J.: Fixed Point Theory. Springer, Berlin (2003)

\section{Submit your manuscript to a SpringerOpen ${ }^{\circ}$ journal and benefit from:}

- Convenient online submission

- Rigorous peer review

- Open access: articles freely available online

- High visibility within the field

Retaining the copyright to your article

Submit your next manuscript at $>$ springeropen.com 